\title{
Wetlands Construídos como alternativas para o tratamento de efluentes em unidades de conservação
}

\section{Arieleen Reis*, María Pilar Serbent, Eduardo Bello Rodrigues}

Universidade do Estado de Santa Catarina. Curso de Engenharia Sanitária. Campus Ibirama. Rua Dr. Getúlio Vargas, 2822. Bairro Bela Vista. Ibirama-SC (CEP 89140-000). *E-mail: arieleen18@hotmail.com.

Resumo. Considerando que as Unidades de Conservação são fundamentais para a conservação da biodiversidade, a presença de sistemas de tratamento de efluentes líquidos é essencial para a integridade destas áreas. No presente trabalho, os sistemas de Wetlands Construídos são indicados como uma alternativa para a realização do tratamento de efluentes líquidos em Unidades de Conservação, devido ao seu baixo custo de implantação e manutenção, o apelo sustentável que esta alternativa de tratamento propõe, além da pouca exigência de elementos construtivos e a harmonização com o ambiente em que é inserido. Neste contexto, o trabalho propõe a utilização do sistema de Wetlands Construídos para o tratamento de efluentes líquidos domésticos na Floresta Nacional de Ibirama em Santa Catarina.

Palavras-chave: Wetlands Construídos, Tratamento de efluentes, Unidades de Conservação, Ibirama.

Abstract. Constructed Wetlands as an alternative for the treatment Recebido: 20/08/2015

Aceito: 28/11/2015

Publicado: 31/12/2015 of effluents in conservation units. Considering that conservation units are paramount for biodiversity, treatment systems of liquid wastes are essential to maintain the integrity of such areas. In this paper, Constructed Wetlands are reported as one viable alternative for liquid wastes treatment systems in Conservation Units, due to low cost implementation and maintenance. These treatment alternatives demand few built-in elements and are developed in harmony into the environment in which it is settled. In this context, the present research proposes the use of Constructed Wetlands for liquid wastes treatment in National Forest of Ibirama.

Keywords: Constructed Wetlands, Protected areas, Wastewater treatment, Ibirama.

\section{Introdução}

De acordo com a Lei $\mathrm{n}^{\circ} 9.985$, de 18 de julho de 2000 (Brasil, 2000), que criou o Sistema Nacional de Unidades de Conservação da Natureza (SNUC), as unidades de conservação (UCs) são espaços territoriais com garantia de proteção, instituídos pelo poder público, que tem como objetivo a conservação da biodiversidade local, ou seja, a proteção e o manejo sustentável da atmosfera, das águas, do solo e subsolo, da fauna e da flora, assim como, de outros recursos naturais existentes.

Para garantir a preservação destas áreas, é obrigatório a elaboração de um documento técnico definido como Plano de Manejo, que auxilia na gestão ambiental, 
além de estabelecer o zoneamento do local, o diagnóstico ambiental da área, os objetivos e as diretrizes para o uso e manejo dos recursos naturais, assim como, as especificações sobre o conjunto de ações e medidas que podem ser realizadas na unidade.

Segundo a Analista Ambiental do ICMBio, Lontra (2015) não há normas e procedimentos específicos sobre 0 saneamento básico nos Planos de Manejo das Unidades de Conservação. Deste modo, estes documentos não seguem um mesmo padrão, e tendo em vista que inúmeras UCs são antigas e foram inauguradas antes da criação de determinadas legislações pertinentes, constata-se um grande déficit em algumas áreas do plano, como é o caso do Saneamento Básico, que é uma ferramenta de extrema importância para garantir o direito a saúde e a conservação do meio ambiente.

Muitas Unidades de Conservação estão em desacordo com as legislações ambientais que dispõem de condições, parâmetros, padrões e diretrizes sobre inúmeras áreas do saneamento, como é o caso do lançamento de efluentes em corpos de água. O tratamento de efluentes ajuda na redução da proliferação de doenças parasitárias e infecciosas, além da diminuição da degradação de solos, corpos hídricos, fauna e flora.

Tendo em vista que a área do saneamento se apresenta de uma forma não específica e descritiva na Lei $n^{\circ}$ 9.985/2000 (SNUC) sugere-se a consideração de soluções alternativas para a realização do tratamento de efluentes nas Unidades de Conservação. Neste contexto, os Wetlands Construídos (WC) surgem como uma opção viável, pois, segundo Philippi et al. (2007) e Sezerino (2006) são sistemas que geram um apelo sustentável, apresentam baixo custo de implantação e operação, possuem eficiência na desinfecção dos esgotos sem a necessidade de aditivos químicos, entram em harmonização com o ambiente em que são inseridos e ainda existe a possibilidade de reuso do efluente tratado.

\section{Wetlands Construídos}

Segundo Ramsar (2010) os Wetlands Naturais ou Zonas Úmidas, são ambientes úmidos naturais, ricos em biodiversidade, que abrangem a extensão de pântanos, charcos e turfas, várzeas, rios, pantanais, estuários, manguezais e até os recifes de corais. Estas áreas regulam o regime hídrico de vastas regiões, além de permitir a recarga de aqüíferos; também dispõem de serviços ecológicos fundamentais para o bem-estar das espécies de fauna e flora.

O sistema de Wetlands Construídos tem como principal objetivo, a melhoria da qualidade da água. Quando aplicados no tratamento de efluentes, o sistema pode ser utilizado como uma alternativa secundária ou terciária, realizando a remoção de nutrientes e reduzindo as taxas de DQO e DBO do efluente (Salati, 2011). Estes sistemas são projetados sob critérios de engenharia e as técnicas de construção variam de acordo com a característica do efluente a ser tratado, da eficiência final desejada na remoção de poluentes, da área disponível para construção do sistema e do interesse paisagístico (Salati et al., 2009; Sezerino, 2006).

Esta metodologia de tratamento se fundamenta na criação de ecossistemas artificiais, com base nos processos naturais de tratamento que ocorrem nos Wetlands Naturais, abrangendo assim, uma complexa variedade de processos físicos, químicos e biológicos que são promovidos pelos elementos constituintes do sistema, ou seja, o material filtrante, os microrganismos e as plantas. As remoções ocorrem principalmente através da filtração e da depuração da matéria orgânica pelos microrganismos formadores do biofilme, que fica aderido ao substrato presente no sistema (Sezerino, 2006; Olijnyk, 2007). Os microrganismos se instalam nas raízes das plantas e no substrato filtrante, onde formam a rizosfera, que segundo Kasuya (2015) é uma zona em que ocorrem diversas alterações microbianas, aumento da atividade e número de organismos, resultando em interações complexas entre as plantas e microrganismos. 


\section{Wetlands Construídos em unidades de conservação \\ Os Wetlands Construídos se} apresentam como uma alternativa coerente com a proposta das Unidades de Conservação, pois utilizam meios naturais de tratamento, exigem poucos elementos construtivos, possuem boas remoções de compostos, como nitrogênio e fósforo, apresentam baixo custo de implantação, operação e manutenção, além de proporcionarem uma harmonia paisagística no ambiente inserido.

Estes sistemas vêm sendo desenvolvidos em áreas de preservação e em parques de diferentes países. Na Tabela 1 a seguir descrevem-se alguns exemplos da aplicação desta metodologia a nível mundial.

Tabela 1. Wetlands Construídos internacionais.

\begin{tabular}{lcccc}
\hline Nome do Parque & Localização & $\begin{array}{c}\text { Tamanho do } \\
\text { Parque }\end{array}$ & $\begin{array}{c}\text { Tamanho do } \\
\text { sistema }\end{array}$ & $\begin{array}{c}\text { Criação do } \\
\text { sistema }\end{array}$ \\
\hline Wakodahatchee Wetlands & Flórida, EUA & $20,23 \mathrm{ha}$ & 0,93 a 4,41 ha** & 1996 \\
France Park's & Logansport, EUA & 40 ha & 0,512 ha & 1999 \\
Tolka Valley Park & Dublin, Irlanda & 50 ha & - & 2000 \\
Ralph Klein Park & Calgary, Canadá & 30 ha & 156 ha* & 2004 \\
Clark County Wetlands Park & Las Vegas, EUA & 1173 ha & - & 2005 \\
Brick Pond Park & North Augusta, EUA & 14,6 ha & 0,6 ha & 2008 \\
Oaklands Park Wetlands & Adelaide, Austrália & - & 2,3 ha & 2015 \\
\hline
\end{tabular}

Fonte: Parks (2004), PARK (2015), Park’s (2015), Otte (2003 apud Openfield Ecological Services), City of Marion (2011b), The North Augusta Riverfront Development Corporation (2007), Hobbs et al. (2003). *O sistema do Ralph Klein Park abrange uma área além das extremidades do parque.

** São 8 leitos que possuem um dimensionamento que varia de 0,93 a 4,41 ha.

No Brasil, os sistemas de Wetlands Construídos já vem sendo utilizados em unidades de conservação e em áreas de preservação de diversas regiões do país, entre elas, pode-se citar o sistema do Parque Ecológico do Tietê em São Paulo-SP, criado em 1988, com o objetivo de demonstrar na prática a real capacidade de depuração do sistema de Wetlands Construídos (Cunha, 2006); o sistema piloto implementado para o tratamento dos efluentes líquidos domiciliares do Parque Nacional do Iguaçu em Foz do Iguaçu-SC (Potratz, 2010); a Estação Ecológica de Carijós, localizada no Município de Florianópolis-SC, que possui um sistema de Wetlands Construídos projetado para 40 pessoas por dia e está em operação desde o ano de 2010 (Thans, 2010); também pode-se citar o sistema do Parque Estadual Fritz Plaumann na Município de ConcórdiaSC, que realizará o tratamento dos efluentes líquidos do parque, e que ainda está em fase de construção (Leão, 2015).
Deste modo, podemos evidenciar o crescimento desta alternativa de tratamento de efluentes em unidades de conservação no país, e embora algumas unidades não apresentem a metodologia de Wetlands Construídos para o tratamento de efluentes, há Planos de Manejo que citam este sistema como um método compatível com as propostas destas áreas. Um exemplo disso é o Relatório da Monitoria do Plano de Manejo da Área de Proteção Ambiental de Cairuçu, no Rio de Janeiro, que apresenta os Wetlands Construídos como uma alternativa para a realização do tratamento de efluentes líquidos na unidade (ICMBio, 2011).

\section{Proposta para a Floresta Nacional de Ibirama}

Este projeto tem como objeto de estudo a Floresta Nacional de Ibirama (Flona Ibirama), que se localiza em Santa Catarina (Figura 1), apresenta uma área de aproximadamente 570 hectares e abrange 


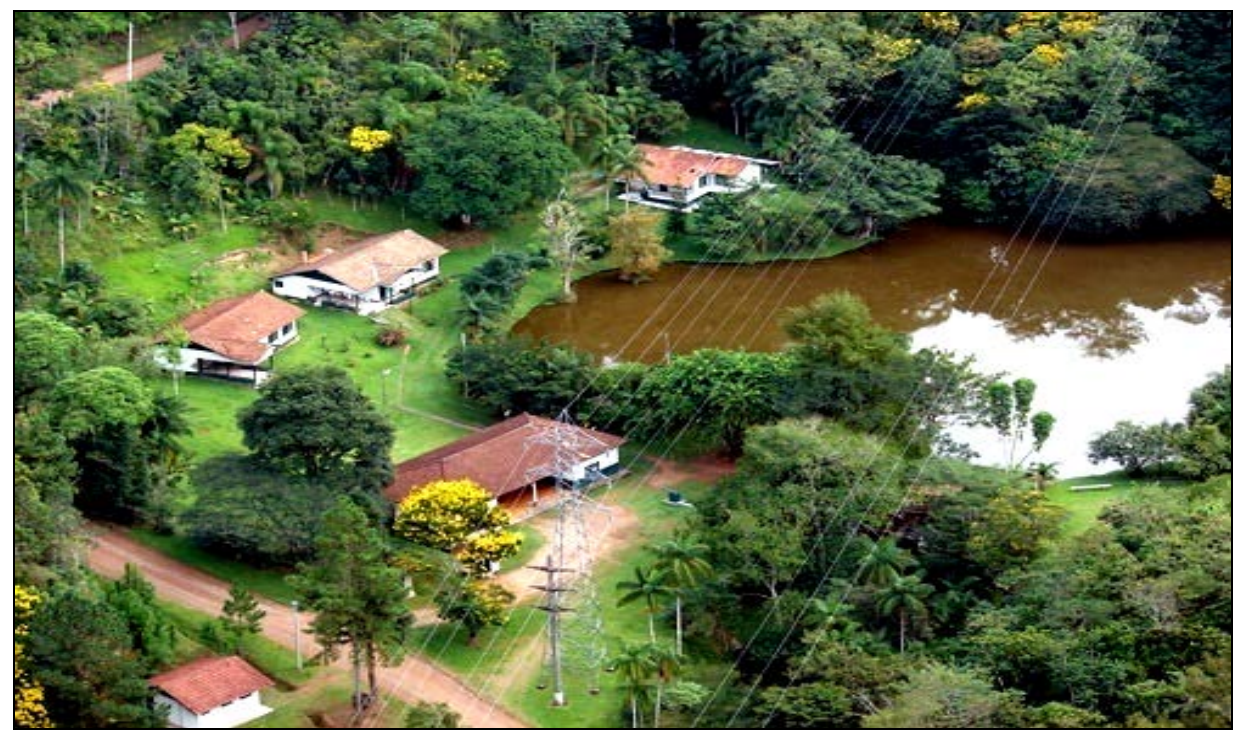

Figura 1. Floresta Nacional de Ibirama, vista área. Fonte: FLONA (2008).

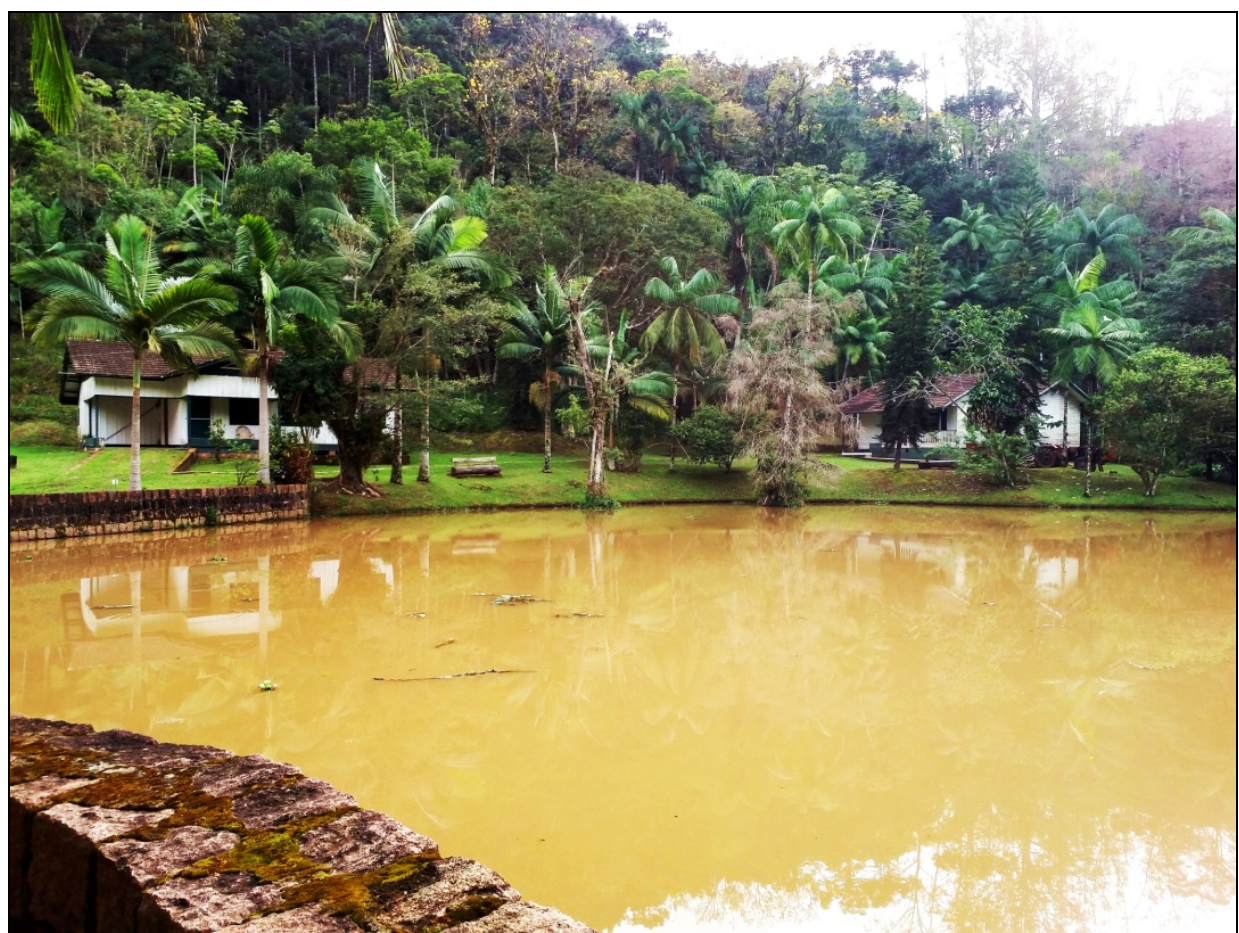

Figura 2. Floresta Nacional de Ibirama.

três municípios do estado, sendo eles Ibirama, Ascurra e Apiúna. A Flona de Ibirama foi criada na década de 1950, em uma parceria entre o estado de Santa Catarina e o Instituto Nacional do Pinho (INP), com o intuito de contribuir com o reflorestamento nas zonas de produção de Pinho.

No início, a unidade era chamada de Horto Florestal de Ibirama, administrado pelo Instituto Nacional do Pinho (INP) e, posteriormente, foi gerido pelo Instituto 
Brasileiro de Desenvolvimento Florestal (IBDF) até 1988, onde com a criação do Decreto Federal n ${ }^{\circ}$ 95.818, em 11 de março de 1988 (Brasil, 1988), a área recebeu uma nova denominação, passando a se chamar Floresta Nacional de Ibirama e uma nova gestão pelo Instituto Brasileiro do Meio Ambiente e dos Recursos Naturais Renováveis (IBAMA), que geriu a unidade até 2007, quando o Instituto Chico Mendes de Conservação da Biodiversidade (ICMBio) assumiu a administração e gestão da Flona Ibirama.

A Floresta Nacional de Ibirama é uma Unidade de Conservação de uso sustentável, ou seja, ela admite que seja realizada a utilização sustentável dos recursos ambientais disponíveis no local, assim como, a presença de visitantes e moradores flutuantes (pesquisadores científicos).

As Florestas Nacionais (Flonas), de acordo com a Lei $n^{\circ} 9.985 / 2000$ (art. 17), devem dar ênfase as pesquisas científicas que focam na presença de métodos de exploração sustentável de florestas nativas, deste modo, com o intuito de aplicar um sistema que vise à integridade da área e que explore o manejo sustentável da unidade, elaborou-se uma proposta de pesquisa científica utilizando o sistema de Wetlands Construídos para a realização do tratamento de efluentes líquidos da Floresta Nacional de Ibirama.

Este projeto possibilita trabalhar educação ambiental com os visitantes da unidade, tendo como público-alvo as escolas e os moradores rurais que vivem no entorno da área, fazendo com que o instrumento principal de divulgação sustentável seja o sistema de Wetlands Construídos. Destaca-se também neste sistema de tratamento, a possibilidade de utilizá-lo como um modelo para a implantação nas propriedades rurais vizinhas.

\section{Material e métodos}

Para que a pesquisa pudesse dar início aos seus trabalhos na sede da Flona Ibirama, foi necessário solicitar uma autorização de pesquisa, que é realizada com o cadastramento do projeto no Sistema de Autorização e Informação em Biodiversidade (Sisbio). Deste modo, ficou liberada a realização de atividades na unidade além de coleta de água, solo e material vegetal para análises.

Inicialmente, fez-se necessário uma pré-análise da área de estudo. Para isso, foram realizadas visitas a campo, préagendadas na sede da ICMBio, que ocorreram entre agosto de 2014 a julho de 2015. Nestas visitas a Unidade de Conservação, foram feitas coletas de solo, identificação da geografia local, assim como, das espécies de plantas nativas que poderiam ser utilizadas no sistema de Wetlands Construídos.

\section{Resultados}

Considerando que ainda não existem normas técnicas brasileiras definindo uma uniformização dos parâmetros e critérios de dimensionamento, este pode ser feito conforme o tipo de efluente, a quantidade de efluente que será tratado (vazão) e a eficiência desejada, entretanto, deve-se sempre projetar a estação a fim de manter a facilidade de operação e manutenção do sistema (Begosso, 2009; Salati, 2011).

O tipo de Wetlands Construído proposto para a Floresta Nacional de Ibirama, foi o Wetland Construído de Fluxo Subsuperficial Horizontal, nesta metodologia o sistema contém pequenas inclinações para o efluente percolar, através de poros que se formam no material filtrante, em um caminho mais ou menos horizontal até alcançar a zona de saída do sistema.

Como proposta de dimensionamento do sistema de Wetlands Construídos para a Flona de Ibirama, levou em consideração a presença de sete servidores fixos por dia, 30 visitantes por semana e três usuários nos alojamentos da unidade. Deste modo, definiu-se o tamanho necessário para a estação de tratamento de $50 \mathrm{~m}^{2}$, sendo $5 \mathrm{~m}$ de comprimento e $10 \mathrm{~m}$ de largura. Os cálculos de dimensionamento do projeto foram feitos para atender aos padrões exigidos pela Resolução 
CONAMA $n^{\circ} 430 / 2011$, sobre lançamento de efluentes em corpos d'água.

Para um melhor funcionamento da estação e visando evitar possíveis colmatações, sugere-se a implantação de dois sistemas em paralelo com alimentação alternada. Molle et al. (2005) define a utilização dos dois sistemas alternados como uma boa opção, pois resulta no aumento da relação área/pessoa, fato que renderiam muitas vantagens, como: a mineralização dos depósitos orgânicos superficiais aportados pelos sólidos em suspensão residuais; a autorregulação da biomassa depuradora e a re-oxigenação dos poros do material filtrante.

Nesta proposta de Wetlands Construídos para a Floresta Nacional de Ibirama, sugere-se a utilização de plantas nativas descritas no Plano de Manejo da unidade. Dentre as plantas que poderiam ser utilizadas no sistema, destacam-se a Taboa (Typha domingensis) e o Junco (Juncus effusus). Estas plantas apresentam alta capacidade depuradora para águas poluídas e são espécies morfológicamente adaptadas para se desenvolverem em sedimentos inundados (Cunha, 2006; Sezerino, 2006; Silva, 2007; Olijnyk, 2007; Salati, 2009; Lima, 2011).

Também se propõe aderir plantas ornamentais ao sistema, do gênero Heliconia, que de acordo com um trabalho realizado por Zanella (2008) apresentam uma grande adaptação e resistência ao sistema de Wetlands Construídos, além de proporcionarem uma estética agradável e atrativa as pessoas que visitam o local.

Como estratégia de avaliação da adequação do projeto serão realizadas análises físico-químicas e microbiológicas no afluente e no efluente tratado. As variáveis usualmente exploradas para a implementação de um Wetland são a demanda bioquímica de oxigênio (DBO), demanda química de oxigênio (DQO), $\mathrm{pH}$, oxigênio dissolvido, nitrogênio total, fósforo total, turbidez, sólidos totais, coliformes termotolerantes e Escherichia coli (E. coli) (Sezerino, 2006; Philippi et al., 2007). Estas variáveis são consideradas importantes para que se defina o nível de eficiência do pré-tratamento, de modo que não venha a comprometer o funcionamento do sistema, principalmente no que se refere à concentração de sólidos no afluente. A realização das análises também são importantes para que se possa avaliar a eficiência da estação de tratamento com Wetlands Construídos.

\section{Conclusões}

A partir deste trabalho visualizou-se a necessidade de aprofundar as discussões e os estudos sobre especificações de sistemas de tratamento de efluentes coerentes com os objetivos das Unidades de Conservação. O avanço na formulação de propostas viáveis é crucial para mobilizar órgãos responsáveis, pois a ausência de informações a respeito do tratamento de efluentes e a falta de detalhamento sobre o saneamento nos Plano de Manejo das Unidades de Conservação indicam a necessidade de estabelecer legislações específicas a respeito destas áreas. A proposta de Wetlands Construídos para o tratamento de efluentes na Floresta Nacional de Ibirama mostra-se viável devido aos benefícios deste tipo de sistema, destacando a sua metodologia adaptável, baixo custo, simples manutenção e operação assim como o atendimento a restrições descritas no Plano de Manejo.

\section{Agradecimentos}

Agradecemos a autorização fornecida pelo SISBio, Número 46035-1 e ao Coordenador Geral da Floresta Nacional de Ibirama por nos permitir a reprodução das imagens da unidade.

\section{Declaração de conflito de interesses}

Os autores declaram não haver conflitos de interesses.

\section{Referências}

Australian Cultural Heritage Management. Kaurna Aboriginal Cultural Heritage Survey for proposed Oaklands Wetland project for City of Marion. Adelaide: 2011. Disponível em: 
$<$ http://dc270.4shared.com/doc/gwS7zbjl/previe w.html>. Acesso em: 14 abr. 2015.

Australian Cultural Heritage Management. Oaklands wetland functionality and landscaping. 2012. Disponível em: $<$ http://www.marion.sa.gov.au/page.aspx?u=812 >. Acesso em: 14 abr. 2015.

Australian Cultural Heritage Management. Oaklands Wetland: Stormwater Reuse Scheme. Adelaide: 2011b. Disponível em: $<$ http://www.marion.sa.gov.au/webdata/resourc es/files/Oaklands_Wetland_Project_Presentatio n.pdf>. Acesso em: 02 nov. 2012.

Begosso, L. Determinação de parâmetros de projeto e critérios para dimensionamento e configuração de Wetlands Construídos para o tratamento de água cinza. Campo Grande: Universidade Federal de Mato Grosso do Sul, 2009. (Dissertação de mestrado em Tecnologias Ambientais).

Brasil. Leis, decretos etc. Decreto $\mathbf{n}^{\circ}$ 95.818, de 11 de março de 1988. Cria a Floresta Nacional de Ibirama, no Estado de Santa Catarina, e dá outras providências. Disponível em: <http://www.planalto.gov.br/ccivil_03/decreto/ 1980-1989/D95818.htm>. Acesso em: 14 abr. 2015.

Brasil. Leis, decretos etc. Lei $\mathbf{n}^{\mathbf{0}} \mathbf{9 . 9 8 5}$, de 18 de julho de 2000. Regulamenta o art. 225, § $1^{\circ}$, incisos I, II, III e VII da Constituição Federal, institui o Sistema Nacional de Unidades de Conservação da Natureza e dá outras providências. Disponível em: <http://www.planalto.gov.br/ccivil_03/LEIS/ L9985.htm>. Acesso em: 14 abr. 2015.

Brasil. Leis, decretos etc. Resolução CONAMA $n^{\circ}$ 430, de 13 de maio de 2011. Dispõe sobre as condições e padrões de lançamento de efluentes, complementa e altera a Resolução ${ }^{\circ}$ 357, de 17 de março de 2005, do Conselho Nacional do Meio AmbienteCONAMA. Disponível em: $<$ http://www.mma.gov.br/port/conama/legiabre. cfm?codlegi=646> . Acesso em: 14 abr. 2015.

Collins, J.; McEntee, D.; Dublin City Council. A constructed wetland for the removal of urban pollution in the Finglaswood Stream, Tolka Valley Park, Dubli. Dublin: Dublin City Council, 2009. Disponível em: $<$ https://www.dublincity.ie/sites/default/files/co ntent//WaterWasteEnvironment/WasteWater/Do cuments/Tolka Valley Park ICW Collins and McEntee Feb 2009.pdf>. Acesso em: 14 abr. 2015.

Cunha, C. A. G. Análise da eficiência de um sistema combinado de alagados construídos na melhoria da qualidade das águas. São Carlos: Universidade de São Paulo, 2006. (Dissertação de Mestrado em Ciências da Engenharia Ambiental).

Flona Ibirama. Plano de Manejo. Ibirama, 2008. Disponível em: <http://www.icmbio.gov.br/ portal/biodiversidade/unidades-deconservacao/biomas-brasileiros/mataatlantica/unidades-de-conservacao-mataatlantica/2223-flona-de-ibirama>. Acesso em: 14 abr. 2015.

IBGE - Instituto Brasileiro de Geografia e Estatística. Síntese dos Indicadores de 2009. 2010. Disponível em: <http://www.ibge.gov.br/ home/estatistica/populacao/trabalhoerendimento /pnad2009/pnad_sintese_2009.pdf>. Acesso em: 4 maio 2015.

ICMBio. Relatório da Monitoria do Plano de Manejo da Área de Proteção Ambiental de Cairuçu. Rio de Janeiro: ICMBio, 2011.

Liènard, A. Wetlands systems for water pollution control. IX. Preface. Water Science \& Technology, v. 51, n. 9, 2005.

Lontra, C. Dúvidas estrutura do Plano de Manejo. [mensagem pessoal]. Mensagem recebida por: <augusta.goncalves@icmbio.gov.br>. Em: 19 jun. 2015.

MMA. Ministério do Meio Ambiente. Roteiro para criação de Unidades de Conservação Municipais. Brasília, 2010. Disponível em: <http://www.ief.mg.gov.br/images/stories/rppn/ roteiro_para_unidades_municipais.pdf $>$. Acesso em: 4 maio 2015.

MMA. Ministério do Meio Ambiente. Áreas Protegidas. 2014. Disponível em: <http://www.mma.gov.br/areas-protegidas/ unidades-de-conservacao/o-que-sao>. Acesso em: 12 dez. 2014.

Olijnyk, D.; Sezerino, P. H.; Soares, A. S.; Philippi, L. S.; Lapolli, F. R. Análise da viabilidade de nitrificação e desnitrificação em sistemas híbridos de filtros plantados com macrófitas (Wetlands). $24^{\circ}$ Congresso Brasileiro de Engenharia Sanitária e Ambiental, 2007.

Openfield Ecological Services. Biodiversity survey of the integrated constructed wetland at Tolka Valley Park, Finglas, Co. Dublin. 2008. Disponível em: <http://www.dublincity.ie/ sites/default/files/content//RecreationandCulture /DublinCityParks/VisitaPark/Documents/Final_ Tolka_Valley_wetland_Biodiversity_survey_20 08.pdf $>$. Acesso em: 19 set. 2012.

Park, Clark Country Wetlands, (Las Vegas). Clark County Wetlands Park - Nature, Close to 
Home.

Disponível

em:

<http://www.clarkcountynv.gov/wetlandspark>. Acesso em: 11 abr. 2015.

Park's, France (Logansport). Constructed Wetlands, 2015. Disponível em: $<$ http://www.francepark.com/index.htm>.

Acesso em: 11 abr. 2015.

Parks, The City of Calgary. Calgary Wetland Conservation Plan. 2004. Calgary, 2004.

Philippi, L. S.; Sezerino, P. H.; Olijnyk, D. P.; Kossatz, B. Eficácia dos sistemas de tratamento de esgoto doméstico e de água para consumo humano utilizando wetlands considerando períodos diferentes de instalação e diferentes substratos e plantas utilizados. Relatório Final. Florianópolis: Universidade Federal de Santa Catarina, 2007.

Philippi, L. S.; Sezerino, P. H. Aplicação de sistemas tipo Wetlands no tratamento de águas residuárias: utilização de filtros plantados com macrófitas. Florianópolis-SC, 2004.

Potratz, V. D. Implantação de um Sistema de Tratamento de Esgoto Domiciliar por Evapotranspiração no Parque Nacional do Iguaçu - Foz do Iguaçu/PR. Foz do Iguaçu: UDC, Faculdade Dinâmica das Cataratas, 2010. (Monografia de graduação em Engenharia Ambiental).

RAMSAR. Secretaria de Biodiversidade e Florestas. Gerência de Biodiversidade Aquática e Recursos Pesqueiros. In: Convenção de Zonas Úmidas, 2010. Disponível em: <http://www.mma.gov.br/estruturas/205/ _arquivos/convencao_ramsar_205.pdf $>$. Acesso em: 12 ago. 2015.

Salati, E. Controle de qualidade da água através de sistemas de Wetlands contruídos. 2011.
Salati, E; Salati Filho, E; Salati, E. Utilização de sistemas de Wetlands construídas para tratamento de águas. Piracicaba: Instituto Terramax, Consultoria e Projetos Ambientais Ltda, 2009.

Sezerino, P. H. Potencialidade dos filtros plantados com macrófitas (Constructed Wetlands) no pós-tratamento de lagoas de estabilização sob condições de clima subtropical. Florianópolis: Universidade Federal de Santa Catarina, 2006. (Tese de Doutorado em Engenharia Sanitária e Ambiental).

Silva, S. C. Wetlands contruídos de fluxo vertical com meio suporte de solo natural modificado no tratamento de esgotos domésticos. Brasília: Universidade de Brasília, 2007. (Tese de doutorado em Tecnologia Ambiental em Recursos Hídricos, Engenharia Civil e Ambiental).

Strickland, T. Brick Pond Park: Restoring Wetlands \& Education “A Perfect Fit”. North Augusta: 2009. Disponível em: $<$ http://www.seswa.org/files/Services/Conferenc e/Past Conferences/2009/12 Brick Pond ParkRestoring Wetlands and Ed [Strickland].pdf>. Acesso em: 17 set. 2012.

Thans, F. Projeto de sistema de tratamento de esgotos sanitários: ICMBio - Reserva de Carijós. Florianópolis: Rotária do Brasil, 2010.

Zanella, L. Plantas ornamentais no póstratamento de efluentes sanitários: Wetlands construídos utilizando brita e bambu como suporte. Campinas: Universidade Estadual de Campinas, 2008. (Tese de Doutorado em Arquitetura e Urbanismo).

Informação da Licença: Este é um artigo Open Access distribuído sob os termos da Licença Creative Commons Attribution, que permite uso irrestrito, distribuição e reprodução em qualquer meio, desde que a obra original seja devidamente citada. 